\title{
Adaptive Mask Coronagraph
}

\author{
P. Bourget ${ }^{1,2}$, R. Vieira Martins ${ }^{2}$, F. Colas ${ }^{3}$, \\ P. Assus ${ }^{4}$ and A. Irbah ${ }^{1}$ \\ ${ }^{1}$ Service d'Aéronomie -CNRS- Verrières le Buisson, France. \\ email: pierre@aerov.jussieu.fr \\ ${ }^{2}$ Observatório Nacional do Rio de Janeiro - MCT - Brazil. \\ ${ }^{3}$ IMCCE, Paris. \\ ${ }^{4}$ Observatoire de Nice -OCA.
}

\begin{abstract}
In this paper, the new coronagraph that will be mounted at the Pic du Midi Observatory (IMCCE - T1m telescope) is presented. To optimize the occulting process of a Lyot coronagraph, a compressed mercury $(\mathrm{Hg})$ drop is used as an occulting disk and its size control offers an adaptation to the seeing conditions or to the Airy diameter fraction needed. In addition to the Hg-mask, a variation on the theme is proposed by a diameter and wavelength adaptive phase mask made of a gas bubble in immersion oil between two optical windows. The instrument concept offers a good versatility to test other mask type and pupil apodization techniques.
\end{abstract}

Keywords. Instrumentation: coronagraph, techniques: interferometry, planets and satellites: astrometry, stars: planetary systems:protoplanetary disks.

\section{Introduction}

The first Hg-mask coronagraph (Bourget et al. 2001) is a Lyot-type coronagraph projected for astrometric observations of faint satellites near Jovian planets and is in work since 2000 at the $1.6 \mathrm{~m}$ telescope of the Pico dos Dias Observatory (LNA - Brazil). The instrument permitted to realize an astrometric survey of faint satellites. The results of systematic astrometric observations of the inner neptunian satellite Proteus (Vieira Martins et al. 2004) have been used for the determination of revised orbital elements (Jacobson et al. 2004). The Hg-mask has been developed to compensate the variations of the apparent diameter of the planets between the observing runs and to simplify the observation mode of the instrument (see figure 1). The new version of the instrument has been developed in 2004 at the Observatório Nacional (ON) of Rio de Janeiro in the framework of a cooperation program between CNPq (Brazil) and CNRS (France). It will be mounted in 2006 at the T1m telescope of the Pic du Midi Observatory (IMCCE). The instrument will be dedicated to observe faint satellites, double asteroids and protoplanetary disks. The integration of the instrument is now realized at the Service d'Aéronomie (CNRS).

In the theoretical case of a stellar image, the occulting disk of a classical Lyot coronagraph had to mask the central core and the first three or four rings in the Airy diffraction pattern to rise good contrast efficiency. If a direct imaging of extrasolar terrestrial planet (ETPs) is desired, the telescope must be able to resolve the orbit of the ETPs away of the fourth diffraction ring and this could only be possible with an extreme large telescope (ELT). Recent works on pupil apodization techniques (Guyon 2003; Aime et al. 2005) dedicated to the high dynamic range imaging and exoplanets detection show that it is possible to obtain a closest higher contrast. The pupil apodization induces a reduction of the point-spread function (PSF) diffraction rings energy. This process applied on a Lyot 

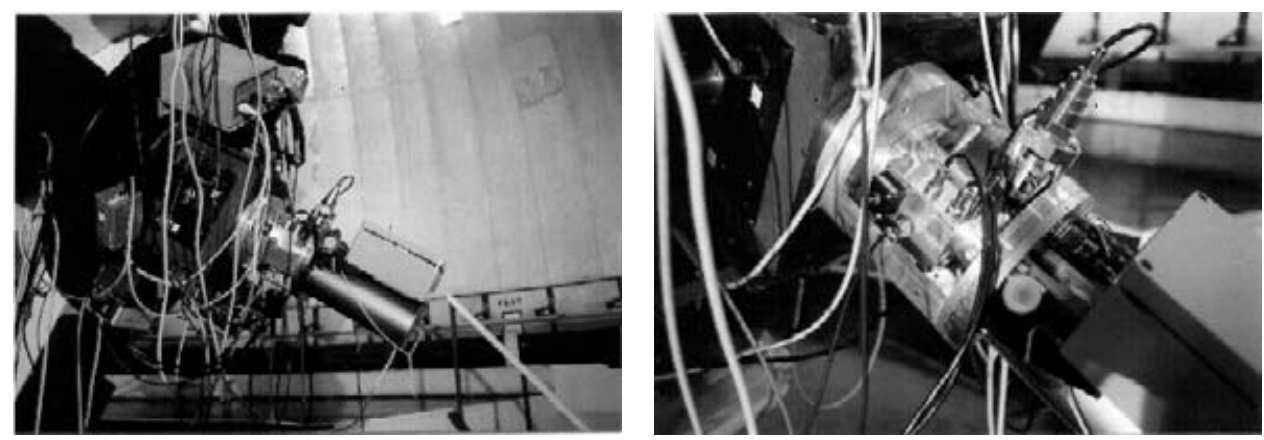

Figure 1. First generation of the Hg-Mask Coronagraph on the $1.6 \mathrm{~m}$ telescope of the LNA-Brazil.

coronagraph does not limit the dynamic angular resolution efficiency to mask the three or four first rings of the PSF.

The phase mask proposed by Roddier \& Roddier (1997), is much smaller than the $\mathrm{Hg}$ mask. It covers only a fraction of the PSF and introduces a 180 degree phase shift on the incoming wave. Even if a pupil apodization (Aime et al. 2002; Guyon \& Roddier 2002; Soummer et al. 2003) can reach a closest full coronagraphic extinction with the phase mask, the instrument is strongly dependent on the phase mask diameter to equalize the of opposite phases amplitudes. A bubble of gas in immersion oil gets a variable diameter phase mask able to be chromatically controlled. The properties of the immersion liquid superficial tension induce the circularity of the phase mask; the diameter control provides the optimization of the nulling efficiency. The main objective of the new instrument is the development of the adaptive mask concept by the means of "no-solid" masks.

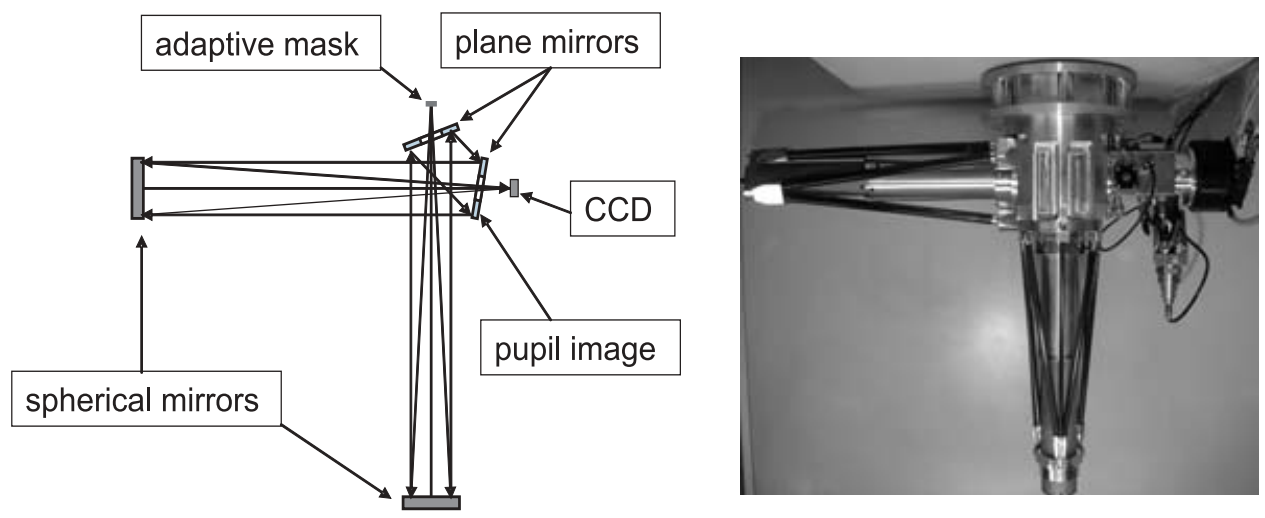

Figure 2. Optical layout of the Coronagraph and mechanical concept.

\section{The instrument}

The optical design of the instrument accepts an incoming beam of focal ratio near f/17. Instead of a big field instrument as the first version of the Hg-mask coronagraph dedicated to the astrometric observations of faint satellites ( Bourget et al. 2004), the instrument presented in this work is a $9 \mathrm{~mm}$ circular field. The optical design is an optical cavity of spherical mirrors. The secondary mirror occultation in the pupil image is used for the light entrance(see figure 2). The occulting disk unit accepts six different adaptive 
masks and offers an XY centering micrometric control. To avoid the mechanical flexion, a carbon-fiber structure is used in the geometrical extension of the instrument. As a first step of apodization, an iris diaphragm is placed on the pupil image plan near the second plane mirror. Both faces of the plane mirrors are polished to accept different kinds of reflection profiles for the amplitude apodization tests on the optical bench.

\section{The adaptive masks}

\subsection{The Hg-Mask}

The use of an ever finite numbers of fixed-diameter occulting disks makes difficult the Lyot coronagraphy. In fact, difficulties appear when changing the disk during observations since it has not often the good size and need to be centered with appropriate offsets. We proposed a solution to this problem by using mercury to realize variable diameter occulting disks. The mercury has often been used in astronomy due to its optical properties. We take into account the high density of the mercury by its superficial tension which allows to make a spherical drop with an optical quality. The drop is glued between optical windows by the van Der Waals forces and is used as the occulting disk (see figure 3 ). In addition, immersion oil is placed to avoid the mercury oxidation and the internal reflection between the windows. The whole system is used as the occulting disk. In a Lyot coronagraphic process the occulting mask acts on the scattered light as all the optical elements. The phase errors produced by the optical roughness of both (optics and mask) induce the scattered light ("Halo"). The minimum of the superficial tension potential function provides a toric free surface of mercury physically perfect $(\lambda / 100)$. The control of the distance between the optical windows acts on the tore diameter with a sub micron-precision. The three dimensional occulting process on the tore induces an apodization of the occultation. The diameter limits are 10 microns to 1.5 millimeters with different ranges of mercury drops compression. The surface of the tore is locally perfect and provides high diffraction quality of the occulting disk. On the optical bench or in the case of adaptive optics, the size control allows the adaptation of the mask to the Airy diameter fraction needed. In the case of a classical telescope, the process allows an adequation to the seeing conditions or to the apparent diameter of a resolved object.



Figure 3. Hg Mask: The distance between the optical window and the pellicle film induces the size control of the toric free surface of mercury. 


\subsection{The adaptive Phase-Mask}

A gas bubble in immersion oil between optical windows is used to obtain a phase retardation of 180 degree on the core of the PSF. The phase delay $\phi$ through the bubble is given by:

$$
\phi=2 \pi \frac{e\left(n-n^{\prime}\right)}{\lambda}
$$

where $n$ and $n$ ' are respectively the refractive index of the liquid and the bubble, $e$ the distance between the optical windows and $\lambda$ the observation wavelength.

The distance parameter $e$ allows to control both the chromatism and the diameter (see figure 4). Typical values for $n$ and $n$ ' are respectively about 1.5 and 1 leading to the delay control expression $e \approx k \lambda$ where $\mathrm{k}$ is an integer. The variation of the retardation is a linear function of $e$ and the diameter is an $e^{-1 / 2}$ function. For an $\mathrm{f} / 17$ telescope (case of the $1 \mathrm{~m}$ telescope of Pic du Midi Observatory), the Airy radius in the visible wavelength ( 0.5 microns) is about 10 microns. A bubble of 15 microns can be spread to obtain a 20 microns phase mask diameter with a 4 microns thickness. To minimize the total flux inside the Lyot stop, the optimum size of the phase mask is done by Roddier \& Roddier (1997) as 0.43 times the radius of the first dark ring in the Airy pattern, which is $0.53 \lambda / \mathrm{D}$. These values are quite different in our case because of the internal reflections in the bubble and the transmission difference through the windows and the immersion oil. The adaptive phase mask allows a direct optimization of the nulling effect to the wavelength range of interest. The parameters to be controlled are $e$ and the phase mask diameter. The original size of the bubble defines the range of the phase mask diameter. The non-linearity dependance between $e$ and the phase mask diameter makes difficult the game of adjustment. To avoid this problem the bubble pressure (and refractive index $n \prime$ ) is another parameter which can be controlled even if depending on the tore diameter for a fixed thickness $e$. The coating used on the optical windows is another parameter to be computed to minimize the wavelength dependence of the delay. As a first step, the adaptive phase mask proposed by the instrument offers a thickness and bubble pressure control. The main technical challenge is the determination of the parameters able to reduce the wavelength dependence of the process preserving the diameter control to equalize the shifted phase amplitudes.
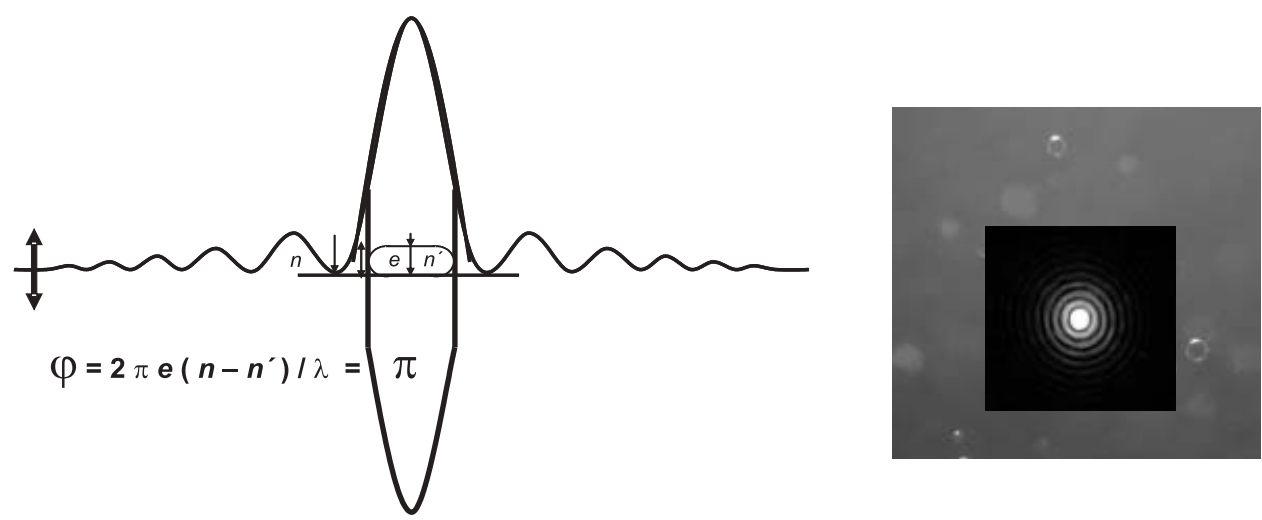

Figure 4. Phase Mask: The gas bubble acts as a phase mask on the core of the PSF, acting on the size control of the bubble we obtain a direct visibility of the nulling efficiency. 




Figure 5. Coronagraph on the optical bench at the Service d'Aéronomie -CNRS. We use a collimator and a $12 \mathrm{~cm}$ Cassegrain telescope to create a PSF on the mask unit.

\section{Conclusions}

The instrument is dedicated to image double asteroids, faint satellites near Jovian planets and protoplanetary disks. The integration of the instrument on the optical bench at the Service d'Aéronomie (CNRS) figure 5 will permit the development of the adaptive phase mask. The instrument will be tested at the T1m telescope of the Pic du Midi Observatory (IMCCE), the optical qualities of the telescope and the $1 \mathrm{~m}$ aperture combined with subarcsecond seeing conditions will allow the quantification of the extinction possibilities of the Adaptive Mask Coronagraph.

\section{Acknowledgements}

We would like to acknowledge the Laboratório Nacional de Astrofísica (LNA-Brazil) for the fellowship that permitted to conclude the first part of the project. The authors thank the CNPq-Brazil for partial support of this work.

\section{References}

Aime, C. 2005 PASP. 117, 1012

Aime, C., Soummer, R., \& Ferrari, A. 2002, A $\mathscr{G} A$. vol. 389, p. 334

Bourget, P., Veiga, C.H., \& Vieira Martins, R. 2001, PASP. 113, 436

Bourget, P., Veiga, C.H., Vieira Martins, R., Assus, P., \& Colas, F. 2004, EAS Publication Series., Astronomy with High Constrast Imaging II, vol. 12, 205

Guyon, O. 2003, A $\& A$., vol. 404, p. 379

Guyon, O. \& Roddier, F. 2002, A $\& A$. vol. 391, p. 379

Jacobson, R.A. \& Owen, W.M. Jr. 2004, AJ. 128, 1412

Roddier, F. \& Roddier, C. 1997, PASP. 109, 815

Soummer, R., Aime, C., \& Falloon, P.E. 2003, A $\& A$. vol. 397, p. 1161

Vieira Martins, R., Veiga, C.H., Bourget, P., Andrei, A.H., \& Descamps, P. 2004, A E์A., vol. 425, p. 1107 

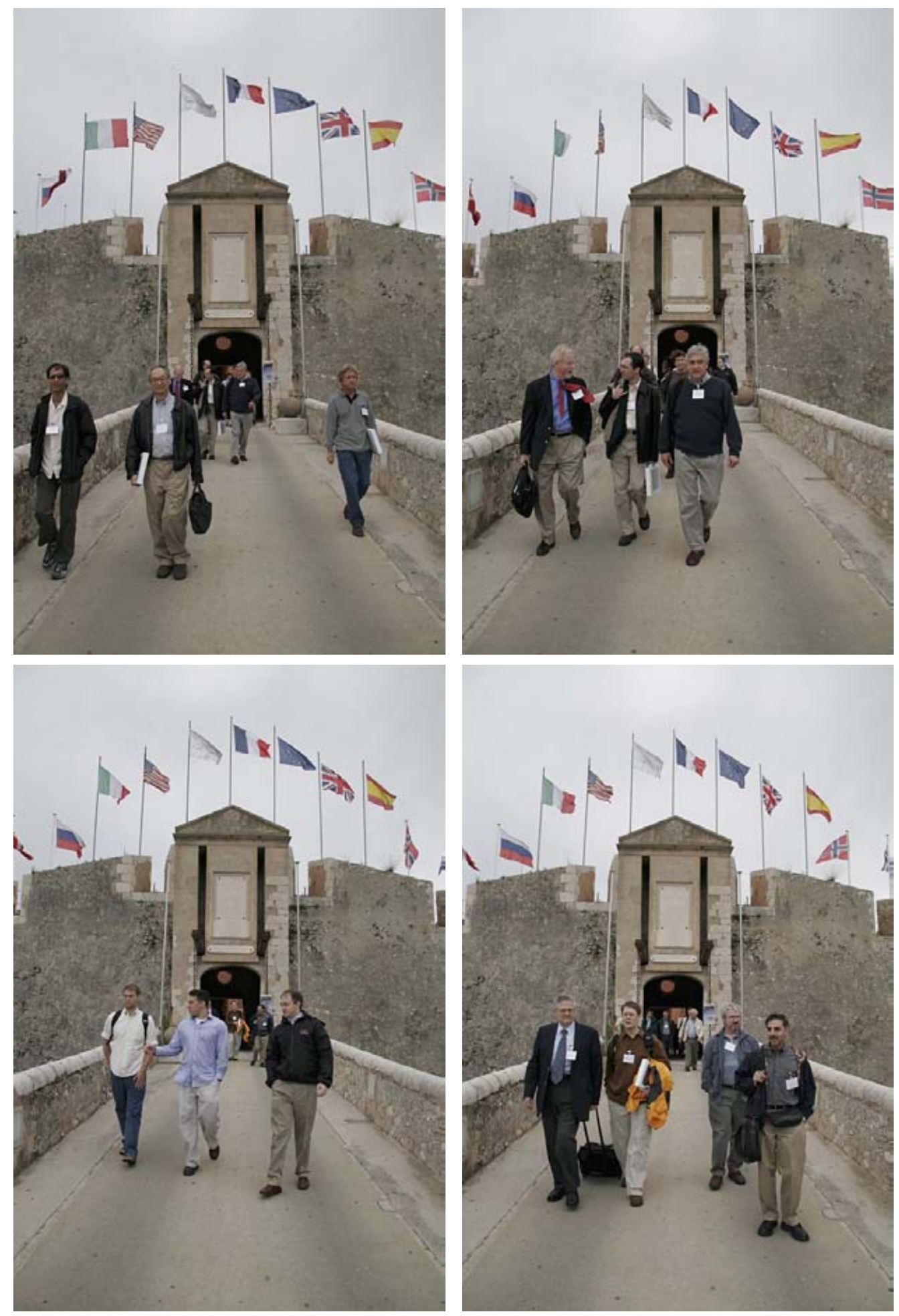

All photographs: Laurent Thareau [1.thareau@free.fr]. 